\title{
DIETARY INTAKES OF AFRICAN AMERICANS BY DIABETES STATUS
}

\author{
A Thesis \\ Presented in Partial Fulfillment of the Requirements for \\ the Degree Master of Science in the Graduate \\ School of The Ohio State University
}

\author{
By \\ Jonathan Matthew Scott, B.S. \\ $* * * * *$ \\ The Ohio State University \\ 2008
}

Master's Examination Committee:

Dr. Christopher A. Taylor, Adviser

Approved by

Dr. Leon McDougle

Dr. Kent P. Schwirian

Adviser

Graduate Program in Allied Medical Professions 



\begin{abstract}
Rates of type 2 diabetes mellitus (diabetes) are disproportionately greater among minority populations in the US, especially among African Americans. Dietary habits are important to the prevention and treatment of diabetes. The dietary intakes of African American adults ( $n=1,622,>17$ years of age) were examined from the 1999-2002 NHANES. Individuals were stratified into three groups (normoglycemia, pre-diabetes, and diabetes) based on: self-reported previous diagnosis; blood glucose; and prescription glucose-lowering medications. Dietary intakes were collected using 24-hour recalls; nutrients and MyPyramid intakes were compared across diabetes status. A total of 1,622 individuals were included in this study. There were statistically significant differences between those who had less than a high school education and those who developed diabetes $(\mathrm{P}<0.001)$. Also those who are married or living as married were more likely to have diabetes $(\mathrm{P}<0.001)$. Lastly, those who had health insurance were more likely to have diabetes $(\mathrm{P}<0.001)$. Those with diabetes were more likely to consume fiber $(\mathrm{P}=0.007)$, cholesterol $(\mathrm{P}=0.012)$, riboflavin $(\mathrm{P}=0.002)$, phosphorus $(\mathrm{P}=0.024)$, potassium $(\mathrm{P}=0.016)$, and sodium $(\mathrm{P}=0.014)$ than those who had normal blood glucose values. Those with diabetes were less likely to consume teaspoon equivalents of added sugars
\end{abstract}


$(\mathrm{P}<0.001)$ compared to those who had normal blood glucose values. These data identify areas for improvement in the dietary habits of African Americans with diabetes and provide valuable information to guide future diabetes education efforts. 


\section{DEDICATION}

Dedicated to my family.

Thanks for all of your love and support 


\section{ACKNOWLDGEMENTS}

I would like to first and foremost thank my advisor, Dr. Chris Taylor, for all of his encouragement, support, and patience throughout my masters program . Without your expertise and time, this thesis would not have been possible, so thank you for being such a motivating mentor and more importantly a great friend.

I would like to personally thank Dr. Kay Wolf for giving me the opportunity to be a part of the combined dietetic internship and masters program here at The Ohio State University. Thank you for such a great educational opportunity, I am greatly honored.

I would also like to thank each member of my thesis panel, Dr.Taylor, Dr.

McDougle, and Dr. Schwirian, for all of your support and input that went into this thesis. I am proud to say that I worked with such a great group of men.

Finally, I would like to give a very special thanks to my family, for being so supportive and motivational throughout my college career. I have gone through some difficult times thus far, and whenever I needed support, you were there for me both emotionally and physically. . And Mom, without you I would not be the person that I am today, so thank you. 
VITA

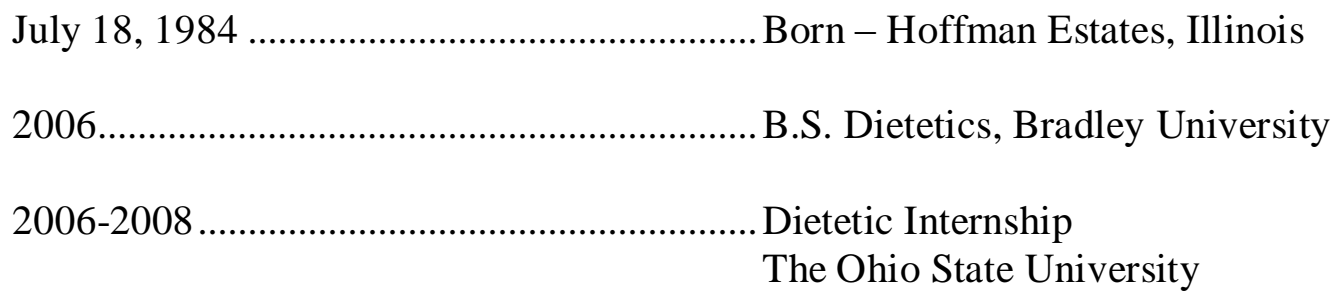

\section{FIELD OF STUDY}

Major Field: Allied Medical Professions 


\section{TABLE OF CONTENTS}

Abstract ...dication

Chapters:

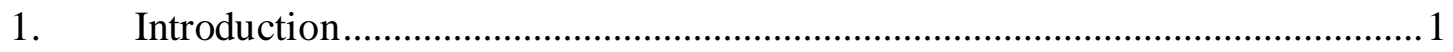

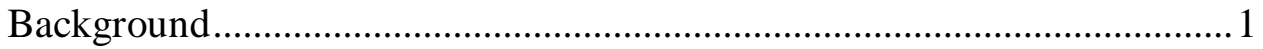

Research Questions ..................................................................................... 3

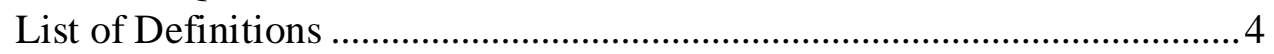

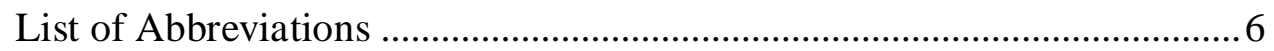

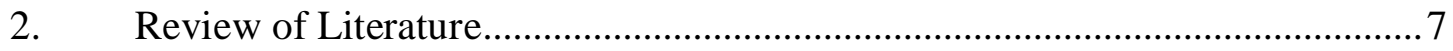

Overview of Diabetes.................................................................................

Etiology of Diabetes.................................................................................

Glycemic Control and Goals ...................................................................11

Diabetes Self-Management Education.........................................................12

Current Dietary Intakes of African Americans without Diabetes ................12

Current Dietary Intakes of African Americans with Diabetes .....................14

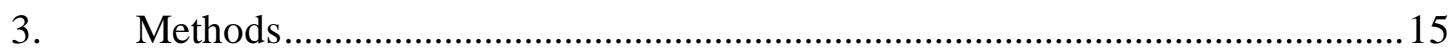

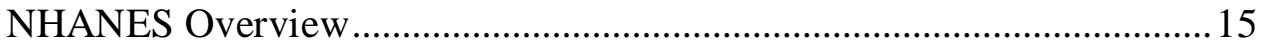

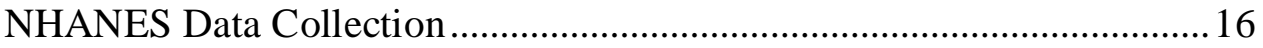

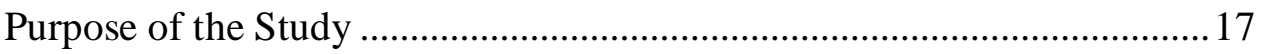

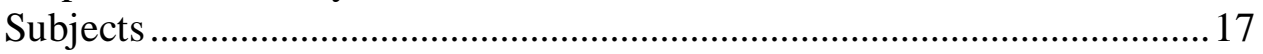

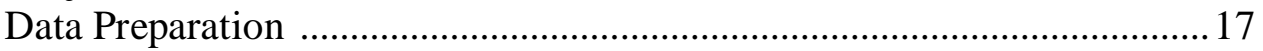

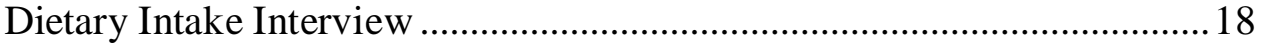

Calculation of Energy Density: ……………………………………......19

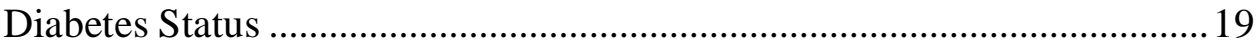




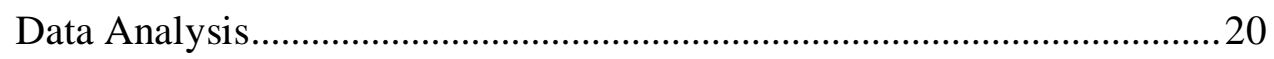

4. Current Dietary Habits of African Americans by Diabetes Status....................22

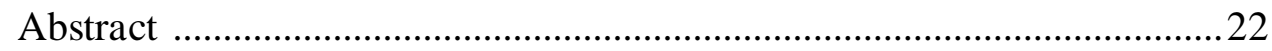

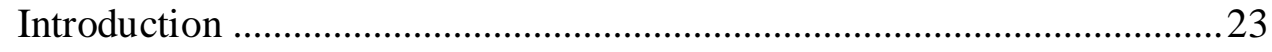

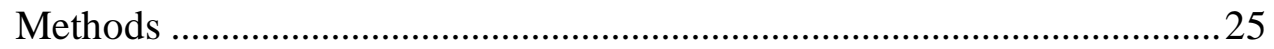

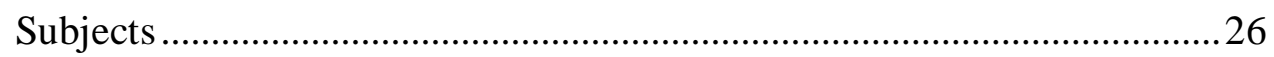

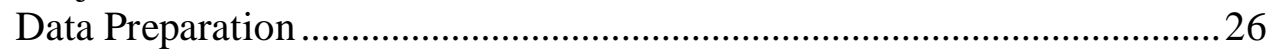

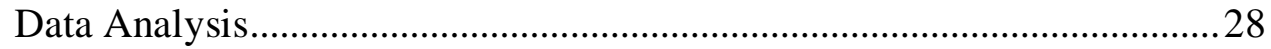

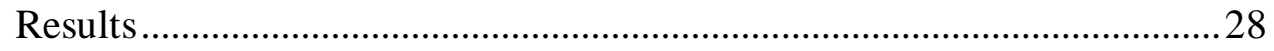

Discussion ...............................................................................................

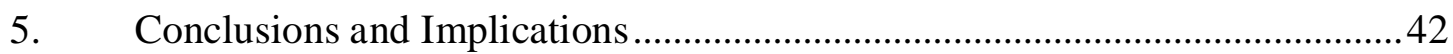

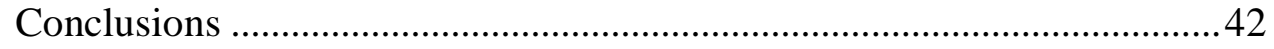

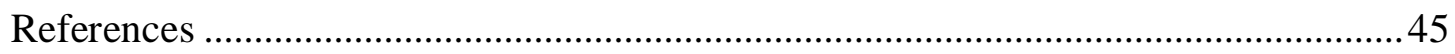




\section{LIST OF TABLES}

$\begin{array}{lll}\text { Table Page } & \text { Pag }\end{array}$

4.1 Differences in Sociodemographics in African American Adults by

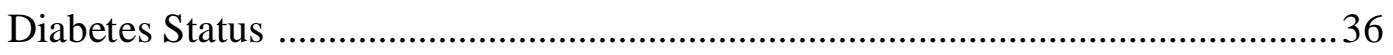

4.2 Mean Differences in Energy-Adjusted Nutrient Intakes of African American

Adults from a 24-hr Dietary Recall by Diabetes Status ........................................38

4.3 Mean Proportions of Recommended Nutrient Intake Levels and the Proportion of African Americans Not Meeting Nutrient Intake Recommendations ....................39 


\section{LIST OF FIGURES}

Figure $\quad$ Page

4.1 Proportion of Energy Derived From Select Macronutrients in African American

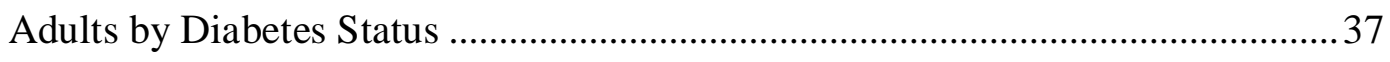

4.2 Energy-Adjusted MyPyramid Intakes (per 1,000 kcals) by Diabetes Status of

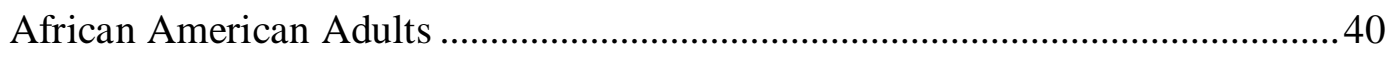

4.3 Proportions of African Americans Meeting MyPyamid Intakes Recommendations

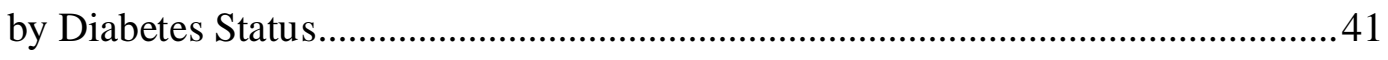




\section{CHAPTER 1}

\section{INTRODUCTION}

\section{Background}

In 2007, the Center for Disease Control and Prevention (CDC) estimated that there were approximately 23.6 million Americans with diabetes, with approximately $90 \%$ as type 2 diabetes (1). Type 2 diabetes effects minority populations disproportionately compared to non-Hispanic Whites (NHW). After adjusting for differences in population age, African Americans (AA) are 1.8 times more likely to develop Type 2 diabetes (diabetes) than NHW (1).

Diabetes is a metabolic disorder that is characterized by an alteration in glucose metabolism resulting from a combination of insulin resistance and insulin deficiency (2). Insulin resistance is described as an inadequate effect of circulating insulin on glucose and fat metabolism. Resistance of insulin causes a state of hyperglycemia, which is responsible for many of the chronic complications of diabetes including blindness, nephropathy, neuropathy and increase risk for cardiovascular disease.

Excess energy intake will produce weight gain; when weight gain translates into obesity, this precipitates insulin-resistance and represents the most 
important risk factor for diabetes (3). The role of obesity in insulin resistance is most strongly correlated to abdominal obesity. In the presence of obesity, dietary recommendations should promote limited energy to foster weight loss and weight maintenance. Treatment of diabetes through diet modification should include structured programs that emphasize a total reduction in energy and fat ( $30 \%$ to total energy) intake (4).

Medical nutrition therapy has the greatest potential for positive clinical outcomes during the initial phases of diabetes when insulin resistance is the greatest. As the disease progresses, glucose-lowering medications and insulin may be needed to complement the lifestyle modifications to achieve euglycemia (4). The primary objective for nutrition therapy for diabetes is to create and implement personalized lifestyle modification strategies that will improve glucose control, improve dyslipidemia and hypertension. The implementation of lifestyle strategies, independent of weight loss, may improve glucose control and other co-morbid conditions (5).

AA have an increased risk for developing diabetes; however, the etiology of this increased risk has not fully been described. One reason may be attributed to lifestyle behaviors and relatively higher rates of obesity (6). Furthermore, little is known about the dietary habits of AAs and the degree to which lifestyle modifications have been adopted for those with diabetes. Understanding the differences in dietary intake habits of African Americans will assist in the development of culturally-appropriate treatment strategies to identify issues relevant to this population. Therefore, the purpose of this study is to identify differences in the sociodemographic profiles of AA adults by diabetes 
status and to identify how the nutrient intake profiles and diet quality of AA adults differ by diabetes status. This is increasingly important because of the potential for lifestyle modifications to play a significant role in weight loss and diabetes prevention.

\section{Research questions}

1. What are the differences in the sociodemographic profiles of African American adults by diabetes status?

2. Are the nutrient intake profiles and diet quality of African American adults different by diabetes status. 


\section{List of Definitions}

Added Sugar- Sugars and syrups that are added to foods during processing or preparation. Added sugars do not include naturally occurring sugars such as those that occur in milk and fruit.

Body Mass Index (BMI)- Used to estimate risk of overweight in adults $\left(\mathrm{kg} / \mathrm{m}^{2}\right)$

Dietary Guidelines for Americans- General guidelines for a healthy diet and decreasing risk of dietary related diseases

Diabetes- A metabolic disorder of carbohydrate metabolism characterized by altered glucose regulation and utilization

Discretionary fats- Fat in a food above the amount that would be found in a lean, lowfat, or fat-free form of the food.

Energy-adjusted- Calories adjusted on a density basis (per 1,000 Calories)

Healthy Eating Index- A measure of diet quality that assesses conformance to federal dietary guidance

Hyperglycemia- An excessive amount of glucose in the blood, generally fasting

$>100 \mathrm{mg} / \mathrm{dl}$ or 2 hour post-prandial $\geq 140 \mathrm{mg} / \mathrm{dl}$

Hyperinsulinemia- An excessive amount of insulin in the blood

Insulin- A hormone released from the $\beta$-cells of the pancreas that enables cells to metabolize and store glucose and other fuels

Insulin resistance- An impaired biological response to either exogenous or endogenous insulin

Isocaloric- Having similar amount of total calories 
MyPyramid- Created by the American Dietetic Association to provide recommendations for Americans on food groups, portion size, variety of nutrients, and physical activity for a healthy lifestyle

NHANES- National Health and Nutrition Examination Survey; a large US nationally representative cross-sectional survey

No Sugar Added- Statement found on products labels, which have no additional sugar has been added by the manufacturer beyond the ingredients of the product.

Obesity- BMI $\geq 30$ in adults and $>95^{\text {th }}$ BMI-for-age percentile in children and adolescents

Overweight- BMI 25-29.9 in adults

Type 2 diabetes- A condition where the body does not produce enough insulin or the cells ignore the insulin

Underweight- BMI $<18.5$ in adults 


\section{List of Abbreviations}

\begin{tabular}{|c|c|}
\hline AA & African American \\
\hline A1c & glycosylated hemoglobin \\
\hline $\mathrm{ADA}$ & American Diabetes Association \\
\hline AI & Adequate Intake \\
\hline BMI & Body Mass Index \\
\hline CADI & Computer-Assisted Dietary Interview \\
\hline $\mathrm{CDC}$ & Center for Disease Control and Prevention \\
\hline CVD & Cardiovascular Disease \\
\hline Diabetes & Type 2 Diabetes Mellitus \\
\hline DPP & Diabetes Prevention Program \\
\hline DRI & Dietary Reference Intake \\
\hline EAR & Estimated Average Requirement \\
\hline FNDDS & Foods and Nutrient Database for Dietary Studies \\
\hline MEC & Mobile Examination Center \\
\hline MNT & Medical Nutrition Therapy \\
\hline NCHS & National Center for Health Statistics \\
\hline NHB & non-Hispanic Blacks \\
\hline NHW & non-Hispanic Whites \\
\hline SES & Socioeconomic Status \\
\hline SMBG & Self-monitoring Blood Glucose \\
\hline USDA & United States Department of Agriculture \\
\hline
\end{tabular}




\section{CHAPTER 2}

\section{REVIEW OF LITERATURE}

\section{Overview of Diabetes}

In 2007, the Center for Disease Control and Prevention (CDC) estimated that there were approximately 23.6 million Americans with diabetes, with approximately $90 \%$ as type 2 diabetes(1). Diabetes has been linked to other co-morbidities such as cardiovascular diseases (CVD), retinopathy, nephropathy, and diabetic foot (7). With diabetes becoming a more inherent problem it is necessary to identify and treat the leading causes of diabetes.

\section{Etiology of diabetes}

Diabetes is a metabolic disorder that is characterized by an alteration in glucose metabolism resulting from a combination of insulin resistance and insulin deficiency (2). Insulin resistance is described as an inadequate effect of circulating insulin on glucose and fat metabolism. Insulin, an endogenous hormone produced by the pancreas in response to elevated blood glucose levels, binds to specific receptor sites located on the plasma membrane of muscle, fat and liver cells. Once insulin is bound to its specific receptor site, a series of 
intracellular protein phosphorylations occur that ultimately lead to the uptake of glucose, fatty acids and amino acids. The insulin molecule and receptor site are normal in most individuals with diabetes; however, several defects have been identified within the intracellular signaling pathways. Insensitivity to insulin results in a hyperglycemic state, which potentiates the production of insulin. This process results in a state of high circulating levels of glucose and insulin. Eventually, insulin resistance leads to a reduction in the ability of insulin to promote glucose uptake in muscle and fat cells and suppression of postprandial liver glucose production $(2 ; 8 ; 9)$.

Excess energy intake will produce weight gain that is linked to insulin-resistance and represents the most import risk factor for diabetes (3). The role of obesity in insulin resistance is most strongly correlated to abdominal obesity. In the presence of obesity, dietary recommendations should limit energy to foster weight loss and weight maintenance. Treatment of diabetes through diet modification should include structured programs that emphasize a total reduction in energy and fat ( $30 \%$ to total energy) intake (4). Modification of dietary components, such as carbohydrate and fat intake, have the potential to impact the degree of insulin resistance and improve glucose tolerance. Bessesen (8) suggested that insulin's capacity to lower blood glucose is improved as total dietary carbohydrate intake is increased, but only to a certain point; however, epidemiologic studies have failed to show a relationship between fructose and sucrose consumption and the development of diabetes. Diets high in dietary fiber appear to be associated with modest beneficial effects on insulin sensitivity and are associated with a decreased risk for developing diabetes (8-10). Small human studies have shown no 
effects on insulin sensitivity when either sucrose or fructose is substituted for isocaloric amounts of starch (9). However, recent research from the Black Women's Health Study indicates that black women who drank $\geq 2$ powered Kool-Aid type drinks, fortified fruit drinks or fruit juices had a $31 \%$ increase risk for developing diabetes. Diet soft drinks, grapefruit juice, and orange juice were not associated with an increase risk for developing diabetes (11)

Diets higher in of dietary fat (35-40\% of total energy intake), specifically saturated fat have been associated with an increase in insulin insensitivity (3). Persistent, long-term intakes of low-fat diets have been associated with improved circulating insulin and glucose concentrations as well as a sustained modest weight loss, $(12 ; 13)$.

The use of MNT as a monotherapy has the greatest positive outcomes during the initial phases of diabetes when insulin resistance is the greatest. As the disease progresses, glucose lowering medications and insulin may be needed to complement the lifestyle modifications to achieve euglycemia. MNT relies on the integration of organized programs that focus on lifestyle change, including education, reduced energy and fat intake, regular physical activity, excess weight 
loss and regular participant contact with a physician (4). Pharmacological therapy interventions begin with oral glucose-lowering medications and progresses to insulin therapy as needed (5).

The primary objective for MNT for diabetes is to create implement personalized lifestyle modification strategies that will improve glucose control, improve dyslipidemia and hypertension. Individuals with diabetes tend to be insulin resistant and overweight and, therefore, MNT should begin with strategies that reduce energy intake and increase energy expenditure through physical activity. The implementation of lifestyle strategies, independent of weight loss, may improve glucose control and other co-morbid conditions. To improve metabolic outcomes, counseling efforts should promote appropriate food choices by using carbohydrate counting, should be encouraged to increase physical activity and should be taught how to monitor blood glucose. These strategies should be implemented promptly after a diagnosis of diabetes or prediabetes (5).

AA have an increased risk for developing diabetes; however, the etiology of this increased risk has not fully been described. One reason may be attributed to lifestyle behaviors and relatively higher rates of obesity (6). Furthermore, little is know about the dietary habits of AAs and the degree to which lifestyle modifications have been adopted for those with diabetes. Understanding the differences in dietary intake habits of African Americans will assist in the development of culturally-appropriate treatment strategies to identify issues relevant to this population. Therefore, the purpose of this study is to identify differences in the sociodemographic profiles of AA adults by diabetes status and 
to identify how the nutrient intake profiles and diet quality of AA adults differ by diabetes status. This is increasingly important because of the potential for lifestyle modifications play a significant role in weight loss and diabetes prevention.

\section{Glycemic Control and Goals}

Glycemic control or self-monitoring of blood glucose (SMBG) is an essential component to diabetes management. SMBG allows patients to take responsibility over their condition and assess whether target goals are being met. Individualized patient goals should dictate the frequency and timing of SMBG. Health care providers should routinely educate and evaluate patients monitoring techniques in order to assure compliance with a SMBG routine.

Goals have been established by the American Diabetes Association (ADA) for glycosylated hemoglobin (A1c), preprandial capillary plasma glucose, and peak postprandial capillary plasma glucose. Postprandial glucose measurements should be taken one to two hours after the beginning of a meal (4).

\begin{tabular}{lc}
\hline Glycemic Control & Goal Range \\
\hline A1C & $<7.0 \%$ \\
Preprandial capillary plasma glucose & $90-130 \mathrm{mg} / \mathrm{dl}$ \\
Peak postprandial capillary plasma glucose & $<180 \mathrm{mg} / \mathrm{dl}$ \\
\hline
\end{tabular}




\section{Diabetes Self-Management Education}

The ADA has recently shifted diabetes self-management education from a didactic approach, which would simply provide patients with information, to a skillsbased approach whereby educators help those with diabetes make better informed selfmanagement decisions (4). Diabetes self-management education has been associated with improved diabetes knowledge, improved self-care behavior and improved clinical outcomes, such as A1c, lower self-reported weight and improved quality of life (4).

The skills that are imperative for diabetes education include SBGM, weight management and lifestyle modification. Key lifestyle modifications to be addressed are promoting healthy dietary habits and increasing physical activity. These lifestyle habits have also been shown to be powerful in the prevention of diabetes in the Diabetes Prevention Program (14); therefore, identifying current lifestyle behaviors provide valuable formative data for diabetes prevention and treatment efforts.

\section{Current Dietary Intakes of African Americans without Diabetes}

Little is known about the current dietary habits of AAs, especially with regard to diabetes status. Jen et al (15) examined the nutrient intakes of inner city AA in Detroit, Michigan using in-person and telephone-based three 24-hr recalls. AA males consumed significantly more energy, fat grams and servings of grain when compared to AA females. AA males reported an average energy intake of 1,590 kcals, while females reported an average energy intake of $1,271 \mathrm{kcals}(\mathrm{P}<0.001)$. The mean fat intake for AA males was $64.5 \mathrm{~g}$ compared to $49.6 \mathrm{~g}$ for females $(\mathrm{P}<0.001)$. Males also consumed significantly more grains (4.73 and 3.93 servings, respectively, $\mathrm{P}<0.001$ ). 
A tracking study completed by Li et al (16) examined the nutrient intakes of inner city AA fourth through seventh graders in Chicago, Illinois. Li et al used the data collected from the two control schools enrolled in the Healthy Eating and Active Lifestyles from school to Home for Kids for analysis. Nutrient analyses from baseline and follow up was collected with a food frequency questionnaire (FFQ) estimated an average of 2,916 kcals, $99.4 \mathrm{~g}$ total fat and $22.6 \mathrm{~g}$ dietary fiber per day at baseline. After a one year follow up the same group of students consumed an average of 2,619 kcals, $88.8 \mathrm{~g}$ total fat, and $19.3 \mathrm{~g}$ dietary fiber.

To explore differences in cultural backgrounds of blacks, Lancaster et al (17) stratified AA from the Third National Health and Nutrition Examination Survey (NHANES III) into four groups: non- Hispanic Blacks born in the US (NHB-US); nonHispanic Blacks born outside the US (NHB-nonUS); Hispanic Blacks born outside the US (HB-nonUS); and Hispanic Blacks born in the US (HB-US). Compared with both groups of Blacks born outside the US, NHB-non-US and HB-nonUS, NHB-US had higher intakes of energy $(1,885,1,854$ and $2,147 \mathrm{kcals}$, respectively) and total fat (71.8, $66.6 \mathrm{~g}$ and $85.1 \mathrm{~g}$, respectively). NHB-US reported lower intakes of carbohydrate compared to both groups of Blacks born outside the US NHB-nonUS and NHB-non-US (248.8, 286 and 298.9 g, respectively). All four groups reported similar protein intakes.

Satia-Abouta et al (18) examined the association between total energy intake and macronutrients with colon cancer risk in AA from North Carolina. Total energy intakes and macronutrient data were estimated from a 100-item semiquantitative Block FFQ. AA had a mean energy intake of $1845 \mathrm{kcals}, 214 \mathrm{~g}$ carbohydrates, $65 \mathrm{~g}$ protein, and $79 \mathrm{~g}$ 
total fat, $26 \mathrm{~g}$ saturated fat, $5 \mathrm{~g}$ alcohol, and $7.4 \mathrm{~g} / 1,000 \mathrm{kcal}$ dietary fiber. Similarly, Diaz et al (19) used the 1999-2000 NHANES data to examine differences in dietary intakes between NHW, AA and Hispanics. AA had a statistically significant higher intake of total energy $(2,119$ kcals $)$ compared to NHW $(2,411$ kcals, $\mathrm{P}<0.05)$. AA also had statistically significant lower total fat intakes compared to NHW (74.6 g and $90.6 \mathrm{~g}$, respectively, $\mathrm{P}<0.05)$. AA reported a mean protein intake of $81.6 \mathrm{~g}$ and $270.1 \mathrm{~g}$ of carbohydrates neither of which were significantly different NHW.

\section{Current Dietary Intakes African Americans with Diabetes}

A validation study conducted by Samuel-Hodge (20) assessed the validity of dietary intake data from AA females with diabetes by comparing reported energy intake with total energy expenditure. Trained interviewers collected a comprehensive list of the previous day's food and drink intake. A series of three 24-hr dietary recalls were collected over the telephone using the multiple pass method. AA females with diabetes consumed a mean energy intake was $1298 \mathrm{kcals}, 151.5 \mathrm{~g}$ carbohydrates, $62.3 \mathrm{~g}$ protein, $51 \mathrm{~g}$ total fat, $15.9 \mathrm{~g}$ saturated fat, $20.2 \mathrm{~g}$ monounsaturated fat, $9.9 \mathrm{~g}$ polyunsaturated fat, and $281 \mathrm{mg}$ cholesterol. 


\section{CHAPTER 3}

\section{METHODS}

\section{NHANES Overview}

Since the 1960's the CDC has conducted a series of health and nutrition surveys to assess the health status of Americans. The National Center for Health Statistics (NCHS), Division of Health Examination Statistics (DHES) has conducted the National Health and Nutrition Examination Surveys (NHANES). The most recent NHANES began in 1999 and has become a continuous national nutrition monitoring and assessment survey $(21 ; 22)$. NHANES is composed of several objectives, which include: to study selected diseases and analyze risk factors for these diseases by monitoring trends in the prevalence, awareness, treatment and control of selected diseases, to study the relationship between diet, nutrition and health, to explore emerging public health issues and new technologies, to establish a national probability sample of genetic material for future research, and to establish and maintain a national probability sample of baseline information on health and nutrition status. The NHANES survey design includes a stratified, multistage probability sample of the civilian non- 
institutionalized USpopulation. NHANES over-samples low-income persons, adolescents, the elderly, pregnant women, African Americans, and Mexican Americans.

There are various stages of sample selection used by NHANES, which included selection of Primary Sampling Units (PSUs). PSUs are counties or small groups of counties, segments within PSUs, which may include a block or group of blocks containing a cluster of households, households within segments, and one or more participants within selected households. A total of 15 PSUs were visited during a twelvemonth period.

\section{NHANES Data Collection}

To begin data collection, hou seholds are identified for inclusion in the NHANES sample. An advanced letter was then mailed to each address informing the occupant(s) that an NHANES interviewer will visit their home. Trained household interviewers visited the home of potential participants, recruit potential participants, and administer screening questionnaires. A computer-assisted personal interview (CAPI) system was used to record the data from the interview. During the home visit, participants were scheduled for the comprehensive health examination at the mobile examination center (MEC) visit.

The health examinations were conducted in the MECs. A small number of survey participant received a health examination in their homes if they are unable to travel to the MEC. There are three MECs that are equipped for use in NHANES, and each MEC consists of four large, inter-connected trailer units. The MEC equipment and data collection systems were checked and calibrated prior to the start of survey data collection 
to ensure quality data. MECs were open five days per week with two examination sessions conducted daily. The examinations required up to three hours to complete.

\section{Purpose of the Study}

African Americans (AA) have an increased risk for developing diabetes. One reason may be attributed to lifestyle behaviors and increased rates of obesity. Understanding the differences in dietary intake habits of AAs will assist in the development of culturally-appropriate treatment strategies to identify issues relevant to this population. Therefore, the purpose of this study is to identify differences in the sociodemographic profiles of AA adults by diabetes status and to identify how the nutrient intake profiles and diet quality of AA adults differ by diabetes status.

\section{Subjects}

NHANES 1999-2002 included 21,004 individuals over the age of 2 months. A nationally representative sample of AAs with dietary intake data, sociodemographic information, completed diabetes questionnaire, and laboratory data were used for analysis. AA were stratified into three categories: normoglycemia; pre-diabetes; or diabetes.

\section{Data Preparation}

The pubic-use data files from NCHS were downloaded from the website and entered into SPSS for preparation and analysis. Some variables required recoding or categorization to provide useful information for the current study. AA were categorized by diabetes status: diabetic, pre-diabetes or normal glycemic using a previous medical history, laboratory values and reported prescription medications. 
Nutrient transformations had to be computed for the following categories: energyadjusted nutrients per 1,000 kcals and percentage of needs met or not met of estimated average requirement (EAR), adequate intakes (AI) and MyPyramid intake recommendations.

\section{Dietary Intake Interview}

During the mobile examination center visits, the dietary interview component was administered to all examinees who agreed to participate. The dietary intake data were used to estimate total intake of energy, nutrients and non-nutrient food components from food and beverages that were consumed 24-hours prior to the interview. Proxy respondents were permitted for survey participants less than six years of age, and assisted interviews were completed with survey participants 6-11 years of age. Trained, bilingual, college-educated dietary interviewers collected all dietary interview data.

Data was collected using an automated data collect process known as the computer-assisted dietary interview (CADI). The CADI interface includes instructions for recoding information about foods, such as brand names and food preparation methods and food amount options. The multiple pass 24-hour dietary interview format was used to collect detailed information about all foods and beverages. The interview passes included a quick list in which respondents were asked to recall all foods and beverages consumed in a 24-hour period the day before the interview, time and place where each food was eaten along with the occasion, details about each food eaten including the amount consumed, and a final review of foods were reviewed with the respondent in 
chronological order. Any additional foods remembered during the process were added to the record as well as modifications for reported foods.

The United States Department of Agriculture (USDA) Foods and Nutrient Database for Dietary Studies (FNDDS) and MyPyramid Equivalents Database were use to code and report the nutrient intake and MyPyramid data respectively, for NHANES 1999-2002. The databases provide nutrient value and MyPyramid estimates for reference 100 gram food portions. Nutrients and MyPyramid data are provided per as the amounts provided by each food as well as cumulative totals per day. Nutrient intakes reported in their respective files do not include those obtained from dietary supplements $(21 ; 22)$.

\section{Calculation of Energy Density}

Due to differences in total caloric intake amongst individuals all macronutrients and micronutrients were converted to a unit of energy density per 1,000 kcals. By standardizing the energy density for each macronutrient and micronutrient comparisons can be more easily made across previously identified stratified groups. The following equation was used to compute energy density:

Energy Density $=($ total nutrient $/$ total kcals $) * 1,000$

\section{Diabetes Status}

Diabetes status will be determined using a three step process using the following criteria:

1. Self-reported diabetes or "border-line" diagnosis by a doctor;

2. Use of prescription medication used to treat hyperglycemia; and

3. Blood glucose levels meeting current diagnostic criteria for diabetes and prediabetes ( $\geq 126$ and $\geq 100 \mathrm{mg} / \mathrm{dL}$ fasting plasma glucose, respectively). 
Diabetes categories will include: normal-glycemic; pre-diabetes; and diabetes. When assessing blood glucose values the total length of food fast prior to blood draw will be evaluated. Those with a blood glucose value indicative of diabetes or pre-diabetes that had a food fast of less than six hours will be considered a false-positive. The exploratory nature of this investigation and the limited number of participants who provided biochemical data from an eight hour fast necessitate the six hour fast to minimize postprandial effects on blood glucose.

\section{Data Analysis}

Differences in dietary intakes and SES by diabetes status will be tested using a one-way ANCOVA and Chi Square. Comparisons in SES factors will be evaluated using the following variables: age; gender; income; education; marital status; health insurance coverage; and food insecurity status. Differences in dietary intakes will be compared across levels of diabetes status. Nutrients targeted include:

- Food energy (kcal)

- Protein (g)

- Carbohydrate $(\mathrm{g})$

- Fat, total (g)

- Alcohol (g)

- Dietary fiber (g)

- Saturated fatty acids(g)

- Monounsaturated fatty acids (g)
- Polyunsaturated fatty acids (g)

- Cholesterol (mg)

- Vitamin A as retinol activity equivalents $(\mu \mathrm{g})$

- Vitamin E as alphatocopherol (mg)

- Vitamin C (mg)

- Thiamin (mg)

- Riboflavin (mg)
- Niacin (mg)

- Vitamin B-6 (mg)

- Folate, total $(\mu \mathrm{g})$

- Calcium (mg)

- Iron (mg)

- Magnesium (mg)

- Phosphorus (mg)

- Potassium (mg)

- Sodium (mg)

- Zinc (mg) 
Nutrient analyses will be compared using energy adjusted values, controlled for SES factors (gender, education, marital status, health insurance and age) identified to differ across diabetes status. Statistical significance will be set a priori at $\leq 0.05$.

SPSS Complex Samples (version 16.0, Chicago, IL) was used to conduct analysis of the NHANES sample. This software allows for the correction of over-sampling of hard-to-reach populations, which results in a nationally-representative sample. When increasing the sample to a national size, SPSS Complex Samples is also essential to provide appropriate standard errors for statistical analyses. 


\title{
CHAPTER 4
}

\section{CURRENT DIETARY HABITS AFRICAN AMERICANS BY DIABETES STATUS}

\begin{abstract}
Introduction

Rates of type 2 diabetes mellitus (diabetes) are disproportionately greater among minority populations in the US, especially among African Americans. Dietary habits are important to the prevention and treatment of diabetes.
\end{abstract}

Methods

The dietary intakes of African American adults ( $n=1,622,>17$ years of age) were examined from the 1999-2002 NHANES. Individuals were stratified into three groups (normoglycemia, pre-diabetes, and diabetes) based on: self-reported previous diagnosis; blood glucose; and prescription glucose-lowering medications. Dietary intakes were collected using 24-hour recalls; nutrients and MyPyramid intakes were compared across diabetes status. 
Results

A total of 1,622 individuals were included in this study. There were statistically significant differences between those who had less than a high school education and those who developed diabetes $(\mathrm{P}<0.001)$. Also those who are married or living as married were more likely to have diabetes $(\mathrm{P}<0.001)$. Lastly, those who had health insurance were more likely to have diabetes $(\mathrm{P}<0.001)$. Those with diabetes were more likely to consume fiber $(\mathrm{P}=0.007)$, cholesterol $(\mathrm{P}=0.012)$, riboflavin $(\mathrm{P}=0.002)$, phosphorus $(\mathrm{P}=0.024)$, potassium $(\mathrm{P}=0.016)$, and sodium $(\mathrm{P}=0.014)$ than those who had normal blood glucose values. Those with diabetes were less likely to consume teaspoon equivalents of added sugars $(\mathrm{P}<0.001)$ compared to those who had normal blood glucose values.

Conclusion

These data identify areas for improvement in the dietary habits of African Americans with diabetes and provide valuable information to guide future diabetes education efforts.

\section{Introduction}

Diabetes rates is a growing public health concern that disproportionately affects minority populations, African Americans (AA) are 1.8 times more likely to develop Type 2 diabetes (diabetes) than non-Hispanic whites (NHW). 
Diabetes is metabolic disorder, which is the result of a combination of insulin resistance and insulin deficiency (2). Insulin resistance is described as an inadequate effect of circulating insulin on glucose and fat metabolism. The insulin molecule and receptor site are normal in most individuals with diabetes, however several defects have been identified within the intracellular signaling pathways. Insulin resistance will lead to a reduction in the ability of insulin to promote glucose uptake in muscle and fat cells and to suppression of postprandial liver glucose production.

Positive caloric intake that leads to weight gain especially related to abdominal adiposity is an important determinant of insulin-resistance and represents the most import risk factor for diabetes (3). Bessesen (8) suggested that insulin's capacity to lower blood glucose is improved as total dietary carbohydrate intake is increased, but only to a certain point. However, epidemiologic studies have failed to show a relationship between fructose and sucrose consumption and the development of diabetes. Diets high in dietary fiber appear to be associated with modest beneficial effects on insulin sensitivity and are associated with a decreased risk for developing diabetes (8-10). Small human studies have shown no effects on insulin sensitivity when either sucrose or fructose is substituted for isocaloric amounts of starch (9). Diets higher in of dietary fat (35-40\% of total energy intake), specifically saturated fat have been associated with an increase in insulin insensitivity (3).

Persistent, long-term intakes of low-fat diets have been associated with improved circulating insulin and glucose concentrations as well as a sustained modest weight loss, $(12 ; 13)$. 
Medical nutrition therapy (MNT) is an effective strategy for prevention and early treatment of type 2 diabetes. MNT relies on the integration of organized programs that focus on lifestyle change, including education, reduced energy and fat intake, regular physical activity, excess weight loss, and regular participant contact with a physician (4). The primary objective for nutrition therapy for diabetes is to create implement personalized lifestyle modification strategies that will improve glucose control, improve dyslipidemias, and hypertension.

AAs have an increased risk for developing diabetes. One reason may be attributed to lifestyle behaviors and increased rates of obesity (6). Understanding the differences in dietary intake habits of African Americans will assist in the development of culturally-appropriate treatment strategies to identify issues relevant to this population. The purpose of this study is to identify differences in the sociodemographic profiles of AA adults by diabetes status and to identify how the nutrient intake profiles and diet quality of AA adults differ by diabetes status.

\section{Methods}

Data from the 1999-2002 National Health and Nutrition Examination Survey (NHANES) were used to examine the dietary habits of African American adults. The NHANES survey design included a stratified, multistage probability sample of the civilian non-institutionalized US population. In home interviews were used to collect sociodemographic data, while physical examination and laboratory data were collected during mobile examination center visits. Hard to reach populations, including lowincome persons, adolescents, the elderly, pregnant women, African Americans, and 
Mexican Americans, were over-sampled to ensure adequate representation for nationallyrepresentative estimates. More information about the design and data collection of NHANES is available elsewhere $(21 ; 22)$.

\section{Subjects}

A nationally representative sample of 1,622 AA adults ( $\geq 18$ years, 797 males and 825 females) were included if they presented dietary intake data, a completed diabetes questionnaire and laboratory data. Women were excluded from the analyses if they were pregnant or lactating at the time of the mobile examination center visit.

\section{Data Preparation}

The pubic-use data files were downloaded from the NCHS website and imported into SPSS for preparation and analysis. Some variables required recoding or categorization to provide useful information for the current study. To assess diabetes status, AA adults were stratified into using a three step process:

1. Self-reported diabetes or "border-line" diagnosis by a doctor;

2. Use of prescription medication used to treat hyperglycemia; and

3. Blood glucose levels meeting current diagnostic criteria $(100-125 \mathrm{mg} / \mathrm{dl}$ for prediabetes, $\geq 126 \mathrm{mg} / \mathrm{dl}$ for diabetes).

Individuals with a blood glucose level greater than $126 \mathrm{mg} / \mathrm{dL}$ but a length of food fast less than 6-hours were considered a false positive for diabetes status. The three groups of diabetes status used for the analyses were normoglycemic, pre-diabetes and diabetes.

The dietary intake data are used to estimate total intake of energy, nutrients and MyPyramid intakes from food and beverages that were consumed 24-hours prior to the 
interview. The multiple pass 24-hour dietary interview format was used by trained interviewers to collect detailed information about all foods and beverages. The US Department of Agriculture (USDA) Foods and Nutrient Database for Dietary Studies (FNDDS) and MyPyramid Equivalents Database were used to code and report the nutrient intake and MyPyramid data, respectively, for NHANES 1999-2002. The databases provided nutrient and MyPyramid estimates for reference 100 gram food portions. Nutrients and MyPyramid data are provided per as the amounts provided by each food as well as cumulative totals per day. Nutrient intakes reported in their respective files do not include those obtained from dietary supplements.

Nutrient transformations were performed to address differences in energy intakes across individuals. Therefore, energy adjusted nutrients and MyPyramid intakes were computed per 1,000 kcals to assess energy-density. The following equation was used to compute energy density:

Energy Density $=($ total nutrient $/$ total kcals $) * 1,000$

By standardizing the energy density for each macronutrient and micronutrient comparisons can be more easily made across previously identified stratified groups.

To identify the proportions of adults meeting current nutrient intake recommendations, nutrient intakes were compare to the Estimated Average Requirement (EAR) or Adequate Intake (AI) values as established by the Dietary Reference Intakes (23-27). The percentages of estimated needs were computed and proportions of adults who did not meet EAR or AI recommendations were compared by diabetes status. 


\section{Data Analysis}

Comparisons in SES factors will be evaluated using the following variables: age; gender; income; education; marital status; health insurance coverage; and food security status. Differences in dietary intakes by diabetes status were tested using a one-way ANCOVA and Chi Square across levels of diabetes status. Nutrient analyses will be compared using energy-adjusted values, controlled for SES factors (gender, education, marital status, health insurance and age) identified to differ across diabetes status. Statistical comparisons were made using log transformed nutrient data to improve normality. Statistical significance will be set a priori at 0.05 .

SPSS Complex Samples (version 16.0, Chicago, IL) was used to conduct analysis of the NHANES sample. This software allows for the correction of over-sampling of hard-to-reach populations, which results in a nationally-representative sample. When increasing the sample to a national size, SPSS Complex Samples is also essential to provide appropriate standard errors for statistical analyses.

\section{Results}

To identify the characteristics related to diabetes in AA adults, differences in sociodemographic factors were examined (Table 1). Women were nearly two-fold more likely to have diabetes $(\mathrm{P}=.03)$. Those with diabetes were significantly more likely to have less than a high school education $(\mathrm{P}<0.001)$, be married or living as married $(\mathrm{P}<0.001)$ or have health insurance $(\mathrm{P}=0.001)$. AA with diabetes $(54.5 \pm 1.0$ years $)$ were significantly older than those with pre-diabetes $(48.7 \pm 1.1)$ or those with no history of diabetes $(39.1 \pm 0.3, \mathrm{P}<.001)$. 
Similar patterns of the proportions of energy obtained from macronutrients were seen across all levels of diabetes status (Figure 4.1). Carbohydrates contributed approximately half of the energy intakes for all levels of diabetes status. AA with diabetes presented with the greatest proportion of energy from total fat (33.5\%) and saturated fat (10.0\%) than those with pre-diabetes (31.9\% and 9.7\%) or normal blood glucose levels (32.1\% and 9.8\%); however, the differences were not significant.

After adjusting for differing sociodemographic factors, there were several significant differences in energy-adjusted nutrients by diabetes status (Table 4.2). Those with diabetes had significantly higher energy-adjusted intakes of fiber $(\mathrm{P}=0.007)$, cholesterol $(\mathrm{P}=0.012)$, monounsaturated fat $(\mathrm{P}=0.024)$, riboflavin $(\mathrm{P}=0.002)$, niacin $(\mathrm{P}=0.047)$, phosphorus $(\mathrm{P}=0.024)$, potassium $(\mathrm{P}=0.016)$ and sodium $(\mathrm{P}=0.014)$ than those who had normal blood glucose values. No significant differences were found for total energy intakes or energy-adjusted macronutrients intakes by diabetes status. Also those with diabetes consumed significantly less energy-adjusted alcohol $(\mathrm{P}=0.046)$ and drinks of alcohol $(\mathrm{P}=0.007)$.

The percent of dietary reference intakes and proportions of AA adults meeting nutrient intake recommendations by diabetes status were examined (Table 4.3). Dietary fiber intakes were low across all levels of diabetes status, with mean intakes representing approximately $60 \%$ of recommended levels. As many as $88 \%$ of AA did not achieve recommended intake levels for dietary fiber. The DRI for total folate was not obtained by as many as $61 \%$ of AA. Approximately $55 \%$ of AA did not consume suggested Vitamin C and Vitamin A intake levels, regardless of diabetes status. Vitamin E, 
calcium, and potassium intakes were low across all levels of diabetes status, with mean intakes representing approximately $50 \%$ of recommended levels. As many as $94 \%, 93 \%$, and $96 \%$ of AA failed to achieve recommended intake levels for Vitamin E, calcium, and potassium on the day food records were collected. On the day of record, $70 \%$ of AA, on average, failed to consume the DRI for magnesium.

After adjusting for differing sociodemographic factors, there were no significant differences in MyPyramid intakes by diabetes status (Figure 4.2). The greatest energyadjusted intakes were found for grains (2.8-3.0 \pm 0.1 oz equivalents) and meat (2.9-3.1 \pm $0.2 \mathrm{oz}$ equivalents). The lowest energy-adjusted intakes were found for milk (0.2-0.3 \pm 0.0 cup equivalents $)$, fruit $(0.5-0.6 \pm 0.1$ cup equivalents $)$, vegetable $(0.7 \pm 0.1$ cup equivalents) and whole grain (0.2-0.3 $\pm 0.0 \mathrm{oz}$ equivalents) intakes, which were below recommended levels for all groups. Whole grains represented only $7.1 \%$ of total grain intake.

The percent of MyPyramid intakes and proportions of AA adults meeting MyPyramid recommendations by diabetes status were examined (Figure 4.3). The recommended MyPyramid intake of grain, vegetables and meat was not obtained by approximately $65 \%, 85 \%$ and $53 \%$ of AA respectively. Fruit and milk intakes were low across all levels of diabetes status, with mean intakes representing approximately $22 \%$ and $50 \%$ of recommended levels respectively. As many as $77 \%$ and $95 \%$ of AA did not achieve recommended intake levels for fruit and milk. 


\section{Discussion}

The foundation of lifestyle modification for individuals with diabetes is dietary modification and the regulation of carbohydrate and fat intakes. These data suggest that little modification in dietary habits is evident from the few significant differences in nutrient and MyPyramid intakes by diabetes status.

The American Diabetes Association (ADA) recommends monitoring carbohydrate intakes through either carbohydrate counting or exchanges as an effective strategy for achieving glycemic control. The ADA also states "with regard to the glycemic effects of carbohydrates, the total amount of carbohydrate in meals or snacks is more important than the source or type" (5). Research has shown that monitoring carbohydrate intakes have been successful at significantly lowering A1C levels (28).

With regard to carbohydrate intakes, AA in this study consumed nearly two-andone-half times the estimated average requirement (EAR) for carbohydrates, regardless of diabetes status. Previous research indicated that when a mean carbohydrate intake exceeds $51 \%$ of total energy intake an unfavorable relationship between higher carbohydrate intake and elevated serum triglycerides levels was observed (29). In our study AA with diabetes consumed $49.6 \%$ of total energy from carbohydrates and those with pre-diabetes consumed $50.9 \%$ of total energy from carbohydrates.

The Diabetes Prevention Program (DPP) was successful in reducing the progression of pre-diabetes to type 2 diabetes through a program that focused on lifestyle modification and reducing fat intake. The DPP (14) recommends limiting total and saturated fat intakes to less than $30 \%$ and $10 \%$ of total energy, respectively. AA with 
diabetes consumed $33.5 \%$ of total energy from fat, while with those with pre-diabetes had the lowest total energy percentage from fat (31.9\%) but still above the DPP recommendations. Those with diabetes also exceeded recommendations for saturated fat intake, $10.0 \%$ of total energy. Prolonged high dietary fat intakes have been linked to the development of obesity and progression of cardiovascular disease, which represents a chronic complication of diabetes.

Recent literature has explored the relationship between dietary fats and risk for developing type 2 diabetes. An elevated ratio of polyunsaturated-to-saturated fat had a protective effect in women, whereas a high intake of total and saturated fat was associated with an increased risk of type 2 diabetes in men. Total fat intake has not always been associated with increased risk in type 2 diabetes however, the fact that total fat reduction was an effective component of diabetes prevention supports a role for total fat intake in enhancing diabetes risk (14). Also, in subjects with diabetes, diets rich in monounsaturated fats ( $\sim 15 \%$ of energy) resulted in increased insulin sensitivity compared to diets high in carbohydrates. Recent literature supports the role of, high-fat diets in the development and potentiation of insulin resistance via multiple mechanisms (30-33). As diet plays a formative role in the progression of diabetes, so too does diet and diabetes have a role in the progression of CVD. A descriptive study by Ma et al (34) was conducted to identify the relationship between changes serum lipids and dietary carbohydrate intake. A significant decrease in high-density lipoprotein (HDL) and increase in the total cholesterol:HDL ratio was positively/negatively related to percentage of calories from carbohydrates. These data suggested that higher total carbohydrate 
intake or percentage of calories from carbohydrate were related to lower HDL and higher serum triglyceride levels. Data from the present study indicated that there are additional ramifications of the current dietary habits of AA, as it has been estimated that approximately $44.6 \%$ and $49.0 \%$ of AA men and women, respectively, over the age of 20 years have CVD (35).

Previous research indicates that the quality of dietary fat also influences insulin sensitivity in humans. Rivellsese et al (36) reported a significantly decrease levels of insulin sensitivity in those who consumed a high saturated fat diet and increased in those who consume a high monounsaturated and $\mathrm{n}-6$ polyunsaturated fat diet. There is also supporting epidemiological evidence that the quality of dietary fat may influence the risk of type 2 diabetes, similarly, saturated fat increasing and unsaturated fat decreasing this risk. Due to differences in fatty acid composition future research should not only assess the distribution of energy through macronutrients, like fat, but also should examine the composition of fatty acids to improve insulin sensitivity and risk for CVD.

Additional dietary habits that represent areas of need were evident from our data. AA also were less likely to meet DRI recommended levels for several minerals crucial to bone health, including calcium, magnesium and folate. Low intakes of these previously mentioned minerals may be due in part to low fruit and vegetable consumption, but also inadequate whole grain and low-fat dairy intake. Previous research suggests that AA adults have lower intakes of milk compare to NHW (37-39). Additionally, AA tend to have lower consumption of fruit and vegetables compared to NHW (40), with the lowest intakes reported among those with limited incomes. Consumption of fruits and 
vegetables have been shown to offer benefits in the prevention and treatment of obesity, diabetes, cardiovascular disease and other chronic diseases (41).

Intakes for many of these nutrients and MyPyramid groups were below recommendations in our data and are linked to bone health. Poor bone health and increased risk for osteoporosis has been linked to diabetes (42). A meta-analysis of type 2 diabetes and risk of fracture found that type 2 diabetes was associated with an increased risk of hip fracture in both men and women $(\mathrm{RR}[95 \% \mathrm{CI}]=2.8[1.2,6.6]$ and 2.1, $[1.6$, 2.7], respectively) (43). These results were consistent between studies of men and women and between studies conducted in the US and Europe. Achemlal et al (42) reported that there was a decreased rate of bone turnover in those with type 2 diabetes, which may result from a decrease in osteoblast mass and function. Also, metabolic abnormalities, which result from poor glycemic control in type 2 diabetes, may contribute to osteopenia. Impaired bone turnover in type 2 diabetes resulted from decreased bone formation and that bone turnover is affected by glycemic control status.

Type 2 diabetes is often characterized by elevated bone mineral density, may impact bone through several mechanisms (44). Obesity, typically present in type 2 diabetes, is strongly associated with higher bone mineral density through mechanical loading and hormonal factors including insulin, estrogen and leptin. Also previous research has indicated that hyperinsulinemia may promote bone formation; however, low levels of insulin may cause reductions in bone mineral density.

Several limitations are inherent with the use of secondary data for the included analyses. The data were collected by the CDC to assess the health status of the US 
population; therefore, data for these analyses were limited to the public-use data files provided. Data were limited to the 24-hr recall to assess current dietary habits in African American adults; however, standardized interview techniques were used by trained interviewers to collect the dietary recalls using the multiple-pass method. The food intakes analyzed represented intakes reported as consumed over the past 24 hours, which may introduce recall bias and may not reflect usual intake patterns. Finally, not all participants in NHANES provided all data of interest. Few participants provided blood glucose data from an 8-hour fast; thus, comparisons across levels of diabetes status may reflect conservative estimates of the link between dietary habits and diabetes status in AA adults.

The lack of significant differences across levels of diabetes status indicates a need for nutrition education for AA with diabetes. Since diet and lifestyle are two key components in diabetes management, it is imperative to create individualized goals with the clients input in mind if outcomes are to be positive. Additionally, the development and implementation of culturally sensitive materials may help to improve diabetes management in AA populations. The role of specific dietary patterns decreased fruit, vegetable, whole grain and dairy intake and increased meat and non-whole grain intake (found in our study to be problematic) are linked to other comorbidities resulting from diabetes. Future research should incorporate the ideas from both the ADA and DPP while at the same time keeping counseling sessions client-centered. Also health care professionals should focus on using culturally appropriate materials when necessary. 


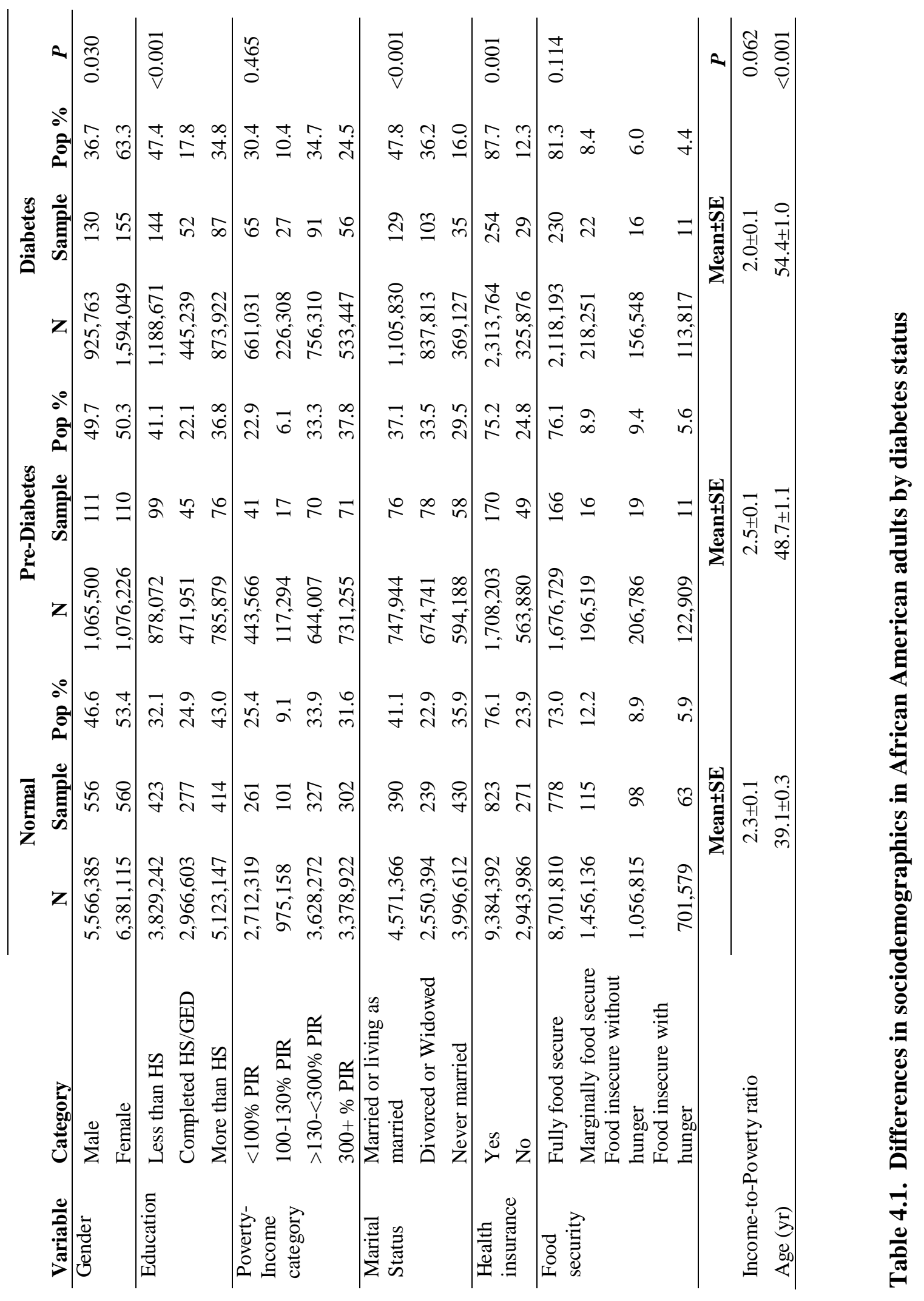




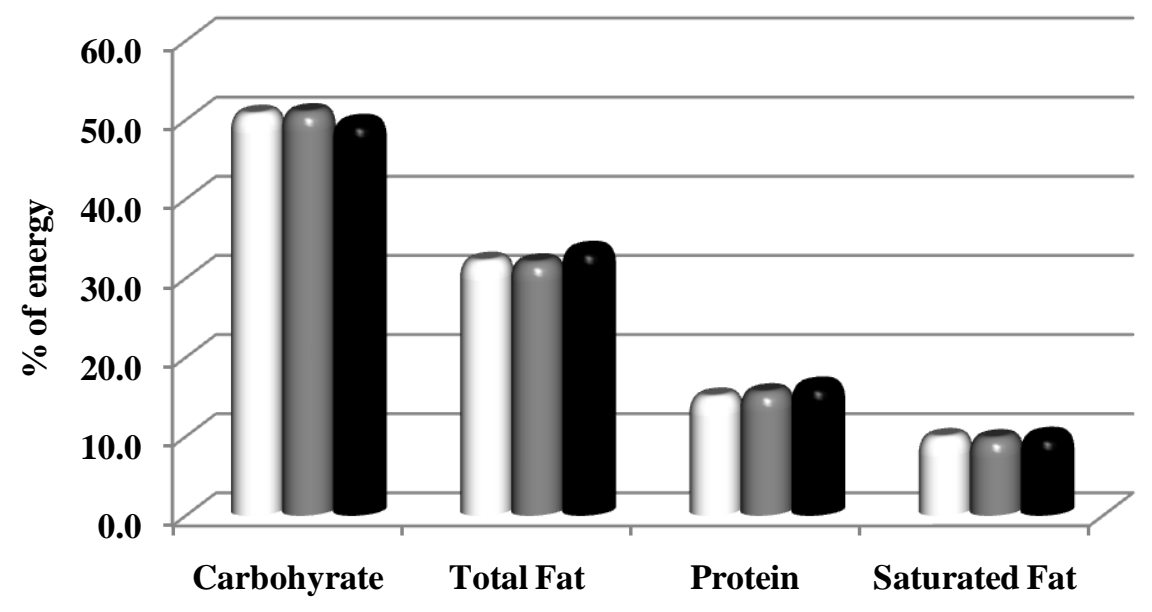

口Normal $\square$ Pre-Diabetes D Diabetes

Figure 4.1: Proportion of energy derived from select macronutrients in African American adults by diabetes status 


\begin{tabular}{|c|c|c|c|c|}
\hline Nurtients ${ }^{1}$ & $\begin{array}{c}\text { Normal } \\
\text { Mean } \pm \text { SE }\end{array}$ & $\begin{array}{c}\text { Pre-Diabetes } \\
\text { Mean } \pm \text { SE }\end{array}$ & $\begin{array}{c}\text { Diabetes } \\
\text { Mean } \pm \text { SE }\end{array}$ & $\mathbf{P}$ \\
\hline Protein $(\mathrm{g})$ & $37.3 \pm 0.5$ & $38 \pm 1$ & $39.5 \pm 1.2$ & 0.055 \\
\hline Carbohydrate (g) & $125.1 \pm 1.2$ & $126.7 \pm 2.1$ & $123.4 \pm 2.4$ & 0.710 \\
\hline Sugars, total (g) & $63 \pm 1.2$ & $64.1 \pm 2.1$ & $60.1 \pm 1.9$ & 0.532 \\
\hline Fiber, total dietary (g) & $6.0 \pm 0.1$ & $6.6 \pm 0.3$ & $6.7 \pm 0.2$ & $<0.001$ \\
\hline Total Fat (g) & $35.8 \pm 0.5$ & $36.1 \pm 0.7$ & $37.4 \pm 0.8$ & 0.069 \\
\hline Saturated fat $(\mathrm{g})$ & $10.9 \pm 0.2$ & $11 \pm 0.3$ & $11.3 \pm 0.3$ & 0.140 \\
\hline Monounsaturated fat (g) & $13.8 \pm 0.2$ & $13.8 \pm 0.3$ & $14.4 \pm 0.3$ & 0.024 \\
\hline Polyunsaturated fat (g) & $8 \pm 0.2$ & $8 \pm 0.3$ & $8.3 \pm 0.4$ & 0.188 \\
\hline Cholesterol (mg) & $142.8 \pm 3$ & $149.7 \pm 10.1$ & $167 \pm 8$ & 0.017 \\
\hline Alcohol (g) & $5.5 \pm 0.6$ & $3.9 \pm 0.8$ & $3 \pm 0.9$ & 0.007 \\
\hline Thiamin (mg) & $0.7 \pm 0$ & $0.7 \pm 0$ & $0.8 \pm 0$ & 0.041 \\
\hline Riboflavin (mg) & $0.9 \pm 0$ & $0.8 \pm 0$ & $1 \pm 0$ & $<0.001$ \\
\hline Niacin (mg) & $11 \pm 0.2$ & $11.1 \pm 0.3$ & $12 \pm 0.4$ & 0.047 \\
\hline Vitamin B-6 (mg) & $0.8 \pm 0$ & $0.8 \pm 0$ & $0.9 \pm 0$ & 0.105 \\
\hline Folate (mcg) & $173 \pm 5.8$ & $168 \pm 7.2$ & $176.7 \pm 7.6$ & 0.690 \\
\hline Vitamin A (mcg RAE) & $323.2 \pm 27.9$ & $301.5 \pm 32.6$ & $335.5 \pm 32.4$ & 0.173 \\
\hline Vitamin E (mg) & $2.9 \pm 0.1$ & $2.9 \pm 0.1$ & $3 \pm 0.2$ & 0.838 \\
\hline Vitamin C (mg) & $53.1 \pm 2.5$ & $53.6 \pm 5.3$ & $54 \pm 4.4$ & 0.296 \\
\hline Calcium (mg) & $290.8 \pm 6.9$ & $287.1 \pm 11.2$ & $306.9 \pm 12$ & 0.200 \\
\hline Iron $(\mathrm{mg})$ & $6.7 \pm 0.1$ & $6.8 \pm 0.3$ & $7.3 \pm 0.3$ & 0.084 \\
\hline Magnesium (mg) & $111.3 \pm 1.6$ & $112.6 \pm 2.9$ & $116.6 \pm 3.7$ & 0.264 \\
\hline Phosphorus (mg) & $525.7 \pm 5.4$ & $531 \pm 10.6$ & $560 \pm 11.8$ & 0.011 \\
\hline Potassium (mg) & $1103 \pm 20$ & $1128 \pm 34$ & $1193 \pm 27$ & 0.015 \\
\hline Sodium (mg) & $1604 \pm 23$ & $1673 \pm 46$ & $1704 \pm 27$ & 0.011 \\
\hline Zinc (mg) & $5.2 \pm 0.2$ & $5.3 \pm 0.3$ & $5.4 \pm 0.3$ & 0.492 \\
\hline Discretionary Oil (g) & $7.5 \pm 0.4$ & $7.4 \pm 0.7$ & $7.6 \pm 1.0$ & 0.162 \\
\hline Discretionary Solid fat (g) & $20.4 \pm 0.3$ & $20.4 \pm 0.9$ & $21.2 \pm 0.7$ & 0.151 \\
\hline Added sugars (tsp) & $11.8 \pm 0.4$ & $11.8 \pm 0.7$ & $10.1 \pm 0.5$ & 0.141 \\
\hline Total drinks of alcohol & $0.4 \pm 0.0$ & $0.3 \pm 0.1$ & $0.2 \pm 0.1$ & 0.007 \\
\hline
\end{tabular}

${ }^{1}$ Nutrients are presented as energy density (nutrients per 1,000 kcals)

Table 4.2: Mean differences in energy-adjusted nutrient intakes of African American adults from a 24-hr dietary recall by diabetes status 


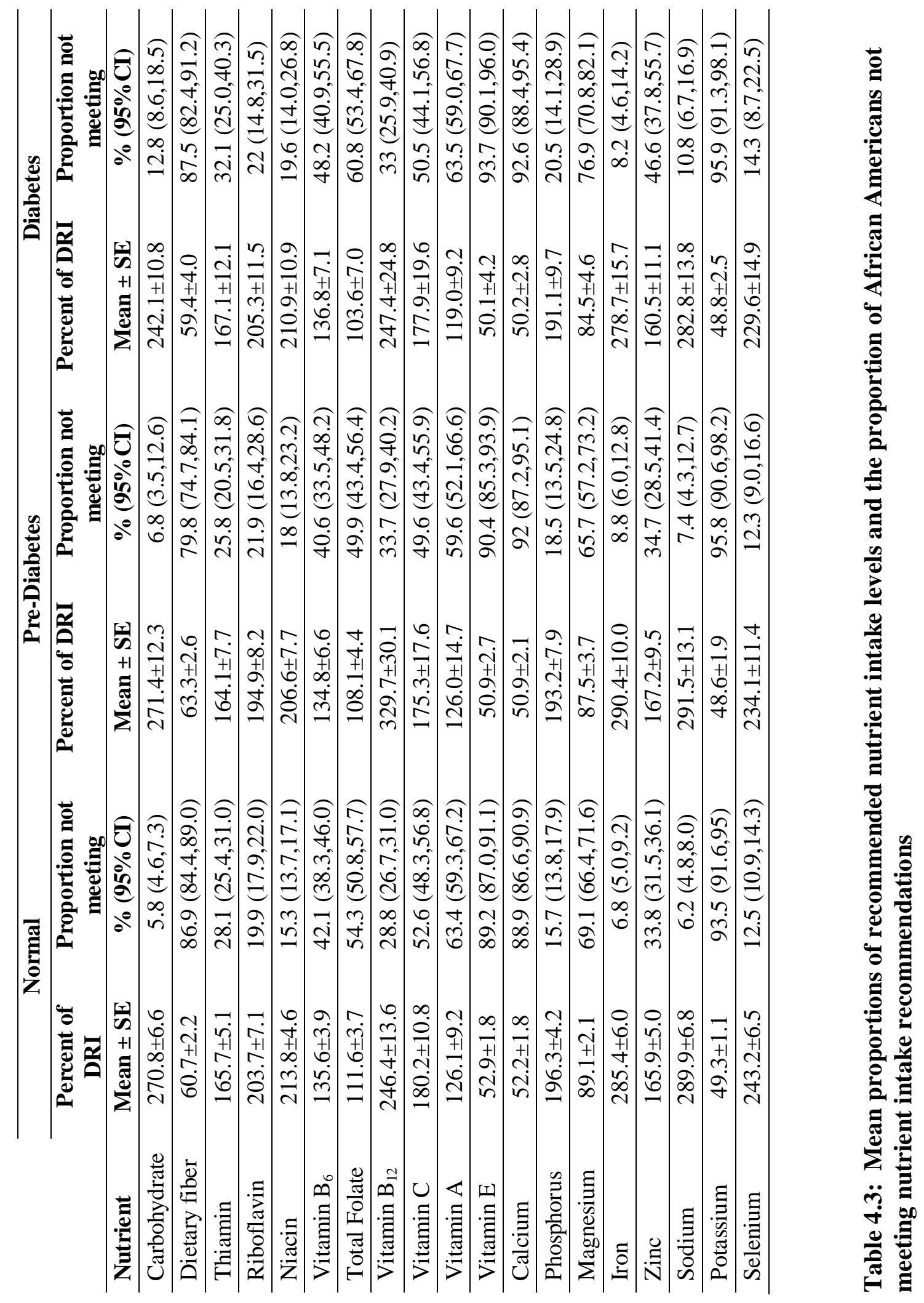




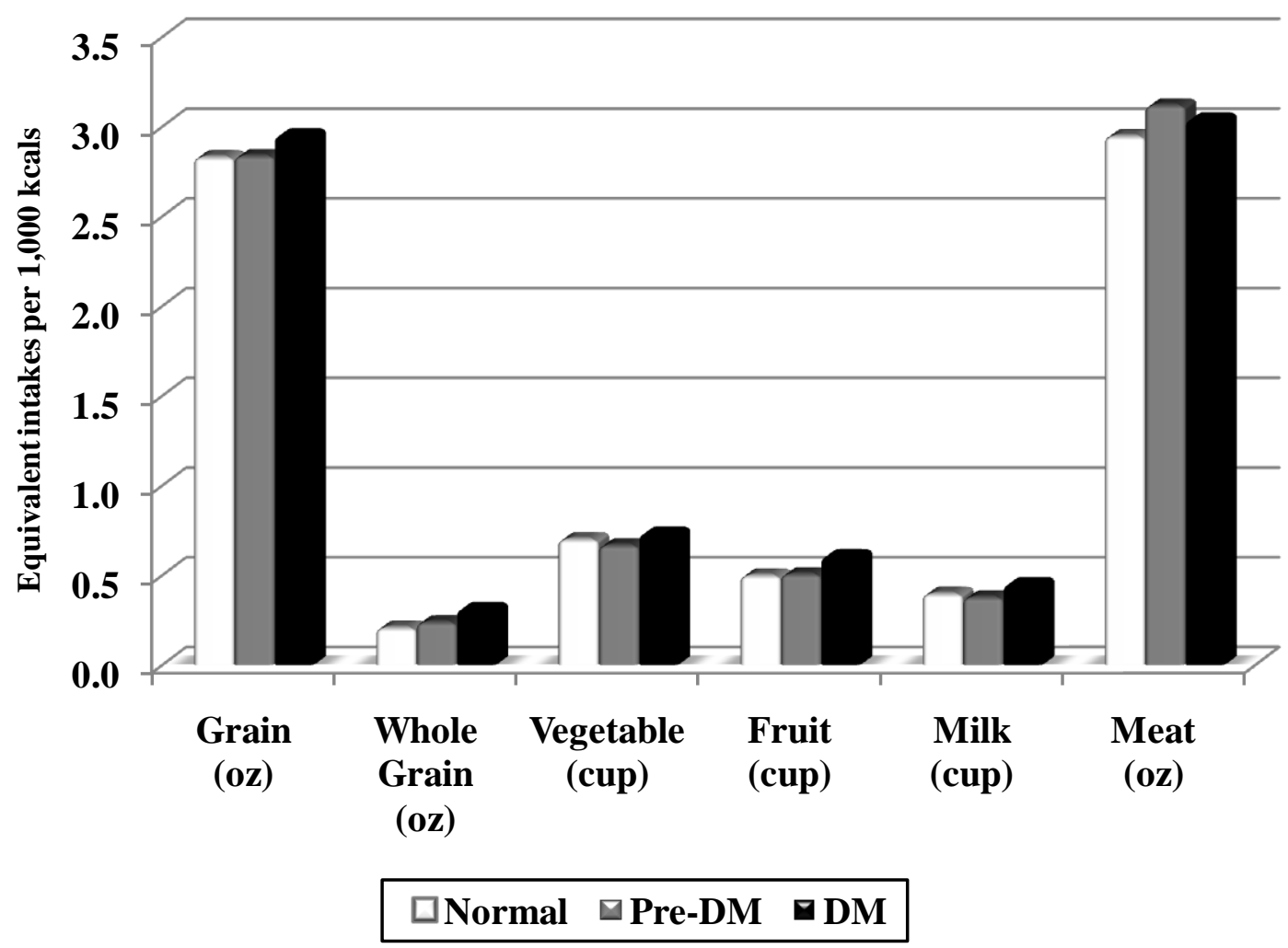

Figure 4.2. Energy-adjusted MyPyramid intakes (per 1,000 kcals) by diabetes status of African American adults 


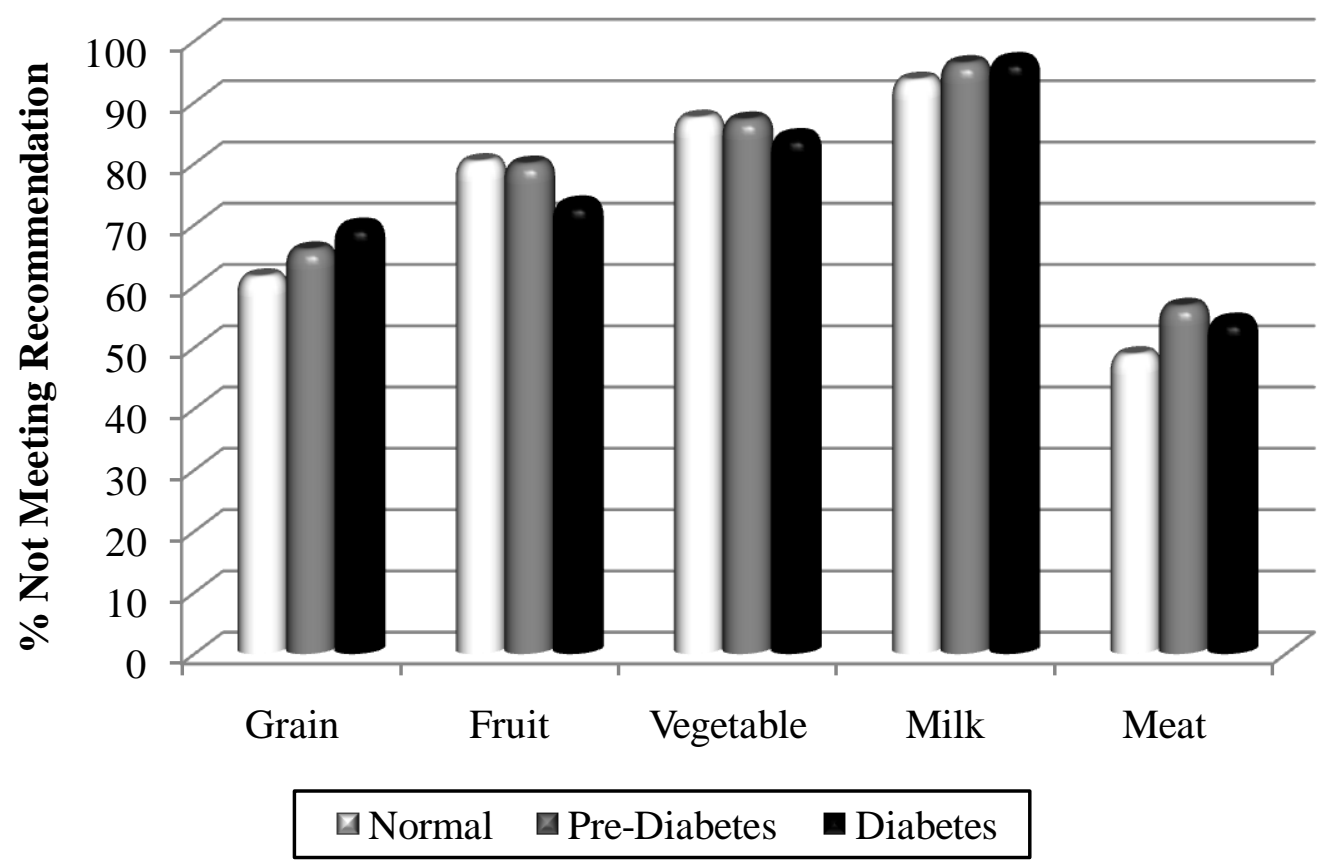

Figure 4.3. Proportions of African Americans meeting MyPyramid intakes recommendations by diabetes status 


\section{CHAPTER 5}

\section{CONCLUSIONS AND IMPLICATIONS}

\section{Conclusions}

These data suggest a need for culturally-specific nutrition education and intervention. Since African Americans (AA) are at a greater risk for developing diabetes than non-Hispanic whites (NHW), health care professionals should focus on implementing appropriate changes in dietary habits that are related to chronic disease risk. Challenges facing health care professionals include socioeconomic issues, a lack of information on AA, and differences in learning styles.

Individuals of lower SES lack the same availability to food resources compared to those of a higher SES. Diet quality plays a prominent role in the prevention and progression of diabetes; however, foods that are less expensive tend to taste better and be higher in calories (45-50). Similarly, food availability is another challenge faced by those of a lower SES. The lack of grocery stores and seasonal markets in areas of lower SES creates barriers to obtaining fresh produce, while the increase in fast food restaurants and convenient stores discourages healthy eating (51). 
Another challenge facing health care professionals is a lack of information on AA. The AA culture is just as diverse as NHW; however, the majority of the research available on AA with diabetes comes from either small local communities or large representative samples such as NHANES. Consequently education materials for NHW are used for AA, which may not specifically address the special needs of this population.

Differences in learning styles may be another challenge facing health care professionals in the treatment of AA with diabetes (52). Learning styles of AA have suggest that AA tend to learn better when in cooperative learning groups. Also instructional techniques that involve more stimulus variety, such as rhythmic and verbal interactions, produce better results as compared to traditional teaching techniques.

\section{Recommendations}

From these data and the current challenges facing health care professionals, it seems appropriate to create a program, which would target low income AA at risk for diabetes and those with diabetes. Specifically the program would target not only healthy eating but also physical activity and social support. The main objective of the program would be to create behavioral modification targeting AA at risk for developing diabetes and those with diabetes. In addition, social support persons should be enrolled in the study. These social support persons along with those with diabetes would: 1) undergo nutrition and physical activity education modeled after the DPP and ADA guidelines and

2) walk for not less than three times weekly for thirty to sixty minutes. In this way both primary prevention of diabetes for friends and family, and secondary prevention of diabetic morbidity for the patient are promoted. 
The AA with diabetes along with their respective social support group members would attend group sessions lead by a lay lifestyle coach to cover lifestyle modification topics detailed by the DPP and ADA curriculum. Classes would convene at common location such as a hospital or YMCA. Both those with diabetes and social support members would receive a pedometer and culturally sensitive dietary training centered on The New Soul Food Cookbook for People with Diabetes. Instruction would also include Healthy Food Choices meal planning using the Soul Food Pyramid, which is an adaptation of the ADA Food Pyramid. 


\section{REFERENCE LIST}

1. Center for Disease Control and Prevention. National Diabetes Fact Sheet, 2007. 9-192008. 8-10-2008.

2. Nolan JJ. What is Type 2 Diabetes? Medicine 2002;30:6-10.

3. Riccardi G, Giacco R, Rivellese AA. Dietary fat, insulin sensitivity and the metabolic syndrome. Clin Nutr 2004;23:447-56.

4. Standards of medical care in diabetes--2007. Diabetes Care 2007;30 Suppl 1:S4-S41.

5. Evidence-based nutrition principles and recommendations for the treatment and prevention of diabetes and related complications. Diabetes Care 2002;25:202-12.

6. The Office of Minority Health. Obesity and African Americans. 9-19-2008. 8-102008.

7. Struijs JN, Baan CA, Schellevis FG, Westert GP, van den Bos GA. Comorbidity in patients with diabetes mellitus: impact on medical health care utilization. BMC Health Serv Res 2006;6:84.

8. Bessesen DH. The role of carbohydrates in insulin resistance. J Nutr 2001;131:2782S$6 \mathrm{~S}$.

9. Daly ME, Vale C, Walker M, Alberti KG, Mathers JC. Dietary carbohydrates and insulin sensitivity: a review of the evidence and clinical implications. Am J Clin Nutr 1997;66:1072-85.

10. Yang EJ, Chung HK, Kim WY, Kerver JM, Song WO. Carbohydrate intake is associated with diet quality and risk factors for cardiovascular disease in U.S. adults: NHANES III. J Am Coll Nutr 2003;22:71-9.

11. Palmer JR, Boggs DA, Krishnan S, Hu FB, Singer M, Rosenberg L. Sugar-sweetened beverages and incidence of type 2 diabetes mellitus in African American women. Arch Intern Med 2008;168:1487-92.

12. Swinburn BA, Metcalf PA, Ley SJ. Long-term (5-year) effects of a reduced-fat diet intervention in individuals with glucose intolerance. Diabetes Care 2001;24:61924. 
13. Carmichael HE, Swinburn BA, Wilson MR. Lower fat intake as a predictor of initial and sustained weight loss in obese subjects consuming an otherwise ad libitum diet. J Am Diet Assoc 1998;98:35-9.

14. Knowler WC, Barrett-Connor E, Fowler SE et al. Reduction in the incidence of type 2 diabetes with lifestyle intervention or metformin. N Engl J Med 2002;346:393 403.

15. Jen KL, Brogan K, Washington OG, Flack JM, Artinian NT. Poor nutrient intake and high obese rate in an urban African American population with hypertension. J Am Coll Nutr 2007;26:57-65.

16. Li J, Wang Y. Tracking of dietary intake patterns is associated with baseline characteristics of urban low-income African-American adolescents. J Nutr 2008;138:94-100.

17. Lancaster KJ, Watts SO, Dixon LB. Dietary intake and risk of coronary heart disease differ among ethnic subgroups of black Americans. J Nutr 2006;136:446-51.

18. Satia-Abouta J, Galanko JA, Potter JD, Ammerman A, Martin CF, Sandler RS. Associations of total energy and macronutrients with colon cancer risk in African Americans and Whites: results from the North Carolina colon cancer study. Am J Epidemiol 2003;158:951-62.

19. Diaz VA, Mainous AG, III, Koopman RJ, Geesey ME. Are ethnic differences in insulin sensitivity explained by variation in carbohydrate intake? Diabetologia 2005;48:1264-8.

20. Samuel-Hodge CD, Fernandez LM, Henriquez-Roldan CF, Johnston LF, Keyserling TC. A comparison of self-reported energy intake with total energy expenditure estimated by accelerometer and basal metabolic rate in African-American women with type 2 diabetes. Diabetes Care 2004;27:663-9.

21. National Center for Health Statistics and Centers for Disease Control and Prevention. The National Health and Examination Survey, 1999-2000. 1999. Hyattsville, MD, US Department of Health and Human Services, Centers for Disease Control and Prevention.

22. National Center for Health Statistics and Centers for Disease Control and Prevention. The National Health and Examination Survey, 2001-2002. 2001. Hyattsville, MD, US Department of Health and Human Services, Centers for Disease Control and Prevention. 
23. Institute of Medicine, Standing Committee on the Scientific Evaluation of Dietary Reference Intakes. Dietary reference intakes for calcium, phosphorus, magnesium, vitamin D, and fluoride. Washington, D.C: National Academy Press, 1997.

24. Institute of Medicine, Food and Nutrition Board. Dietary Reference Intakes for thiamin, riboflavin, niacin, vitamin B6, folate, vitamin B12, pantothenic acid, biotin, and choline. Washington, D.C: National Academy Press, 1998.

25. Institute of Medicine, Food and Nutrition Board. Dietary reference intakes for vitamin C, vitamin E, selenium, and carotenoids. Washington, D.C: National Academy Press, 2000.

26. Institute of Medicine, Food and Nutrition Board. Dietary Reference Intakes for vitamin $\mathrm{A}$, vitamin $\mathrm{K}$, arsenic, boron, chromium, copper, iodine, iron, manganese, molybdenum, nickel, silicon, vanadium, and zinc. Washington, D.C: National Academy Press, 2001.

27. Institute of Medicine, Food and Nutrition Board. Dietary Reference Intakes for energy, carbohydrate, fiber, fat, fatty acids, cholesterol, protein, and amino acids (Macronutrients). Washington, D.C: National Academy Press, 2002.

28. Bergenstal RM, Johnson M, Powers MA et al. Adjust to target in type 2 diabetes: comparison of a simple algorithm with carbohydrate counting for adjustment of mealtime insulin glulisine. Diabetes Care 2008;31:1305-10.

29. Ma Y, Olendzki BC, Hafner AR et al. Low-carbohydrate and high-fat intake among adult patients with poorly controlled type 2 diabetes mellitus. Nutrition 2006;22:1129-36.

30. Hu FB, Manson JE, Stampfer MJ et al. Diet, lifestyle, and the risk of type 2 diabetes mellitus in women. N Engl J Med 2001;345:790-7.

31. Hu FB, Van Dam RM, Liu S. Diet and risk of Type II diabetes: the role of types of fat and carbohydrate. Diabetologia 2001;44:805-17.

32. Van Dam RM, Rimm EB, Willett WC, Stampfer MJ, Hu FB. Dietary patterns and risk for type 2 diabetes mellitus in U.S. men. Ann Intern Med 2002;136:201-9.

33. Van Dam RM, Willett WC, Rimm EB, Stampfer MJ, Hu FB. Dietary fat and meat intake in relation to risk of type 2 diabetes in men. Diabetes Care 2002;25:417-24.

34. Ma Y, Li Y, Chiriboga DE et al. Association between carbohydrate intake and serum lipids. J Am Coll Nutr 2006;25:155-63. 
35. The American Heart Association. Statistical Fact Sheet- Populations 2008 Update. 919-2008. 8-10-2008.

36. Rivellese AA, Lilli S. Quality of dietary fatty acids, insulin sensitivity and type 2 diabetes. Biomed Pharmacother 2003;57:84-7.

37. Storey ML, Forshee RA, Anderson PA. Beverage consumption in the US population. J Am Diet Assoc 2006;106:1992-2000.

38. Robb C, Reynolds L, Abdel-Ghany M. Consumer Preference Among Fluid Milks: Low-Fat vs. High-Fat Milk Consumption in the United States. International Journal of Consumer Studies 2006;31:90-4.

39. Fulgoni V, III, Nicholls J, Reed A et al. Dairy consumption and related nutrient intake in African-American adults and children in the United States: continuing survey of food intakes by individuals 1994-1996, 1998, and the National Health And Nutrition Examination Survey 1999-2000. J Am Diet Assoc 2007;107:256-64.

40. Casagrande SS, Wang Y, Anderson C, Gary TL. Have Americans increased their fruit and vegetable intake? The trends between 1988 and 2002. Am J Prev Med 2007;32:257-63.

41. US Department of Health and Human Services, US Department of Agriculture, US Dietary Guidelines Advisory Committee. Dietary Guidelines for Americans, 2005. Washington, D.C: G.P.O, 2005.

42. Achemlal L, Tellal S, Rkiouak F et al. Bone metabolism in male patients with type 2 diabetes. Clin Rheumatol 2005;24:493-6.

43. Janghorbani M, Van Dam RM, Willett WC, Hu FB. Systematic review of type 1 and type 2 diabetes mellitus and risk of fracture. Am J Epidemiol 2007;166:495-505.

44. Schwartz AV. Diabetes Mellitus: Does it Affect Bone? Calcif Tissue Int 2003;73:515-9.

45. American Diabetes Association position statement: evidence-based nutrition principles and recommendations for the treatment and prevention of diabetes and related complications. J Am Diet Assoc 2002;102:109-18.

46. Drewnowski A. Fat and sugar: an economic analysis. J Nutr 2003;133:838S-40S.

47. Drewnowski A. Obesity and the food environment: dietary energy density and diet costs. Am J Prev Med 2004;27:154-62.

48. Drewnowski A, Specter SE. Poverty and obesity: the role of energy density and energy costs. Am J Clin Nutr 2004;79:6-16. 
49. Drewnowski A, Darmon N. The economics of obesity: dietary energy density and energy cost. Am J Clin Nutr 2005;82:265S-73S.

50. Drewnowski A. The real contribution of added sugars and fats to obesity. Epidemiol Rev 2007;29:160-71.

51. Powell LM, Slater S, Mirtcheva D, Bao Y, Chaloupka FJ. Food store availability and neighborhood characteristics in the United States. Prev Med 2007;44:189-95.

52. Willis MG. Learning Styles of African American Children: A Review of the Literature and Interventions. The Journal of Black Psychology 1989;16:47-65. 